\title{
Error Estimates from Noise Samples for Iterative Algorithm in Shift-Invariant Signal Spaces
}

\author{
Jun Xian \\ Department of Mathematics, Sun Yat-Sen University, Guangzhou 510275, China \\ Correspondence should be addressed to Jun Xian, xianjun@mail.sysu.edu.cn \\ Received 1 September 2010; Accepted 4 October 2010 \\ Academic Editor: Douglas Robert Anderson
}

Copyright (C) 2010 Jun Xian. This is an open access article distributed under the Creative Commons Attribution License, which permits unrestricted use, distribution, and reproduction in any medium, provided the original work is properly cited.

\begin{abstract}
We consider error estimates of iterative algorithm in shift-invariant signal spaces. For the classical sampling and reconstruction algorithm, error estimate from its samples corrupted by white noises are widely studied, but the error analysis of noise with time jitter and iterative noise has not been given as much attention. In this paper, three types of error estimates are studied. In detail, we obtain the error estimate for reconstructing a signal from its noise samples, noise samples with time jitter, and iterative noise.
\end{abstract}

\section{Introduction}

The famous Shannon sampling theorem [1] successful resolves the reconstruction of a function $f$ on $\mathbb{R}^{d}$ from its samples $\left\{f\left(x_{j}\right): j \in J\right\}$, where $J$ is a countable index set. This is a common task in many applications in signal or image processing. The Shannon sampling theorem says that if $f$ is the bandlimited signal of finite energy, then it is completely characterized by its samples. In many engineering applications, such as MRI imaging, signals and images are not band limited. One such example is the shift-invariant spaces. Nonuniform sampling and reconstruction problems in shift-invariant spaces are a relatively recent and active research field [2-12]. The shift-invariant space model developed in the 1990s is successful for many engineering problems, where the signal $f$ to be reconstructed is assumed to live in a shift-invariant space. It has been shown to be suitable and realistic, especially for taking into account of realistic environment, for modeling signals with smooth spectrum, or for numerical implementation $[8,10,12]$.

In this paper, we will assume that the functions or signals all belong to shift-invariant space of the form [2-12]

$$
V^{2}=\left\{\sum_{k \in Z} c_{k} \varphi(\cdot-k):\left\{c_{k}\right\} \in \ell^{2}\right\} .
$$


Many reconstruction algorithms are studied in shift-invariant spaces. For example, the iterative algorithm is obtained in shift-invariant spaces [3]. However, the reconstructing a function from data corrupted by noise has not been given as much attention. Smale and Zhou reconstruct signals from noisy data in [7] and give error estimates for the reconstructed signal [8]. Aldroubi et al. discussed error analysis of frame reconstruction from noisy samples in [2]. Chen et al. gave the estimate of aliasing error for reconstruction algorithm in shift-invariant spaces [4]. We will study error analysis of the iterative reconstruction algorithm from noisy samples in shift-invariant spaces.

In this paper, the following three types of errors are considered for the iterative algorithm in shift-invariant spaces.

(1) Signal samples are affected by some additive noise and are therefore given by

$$
f\left(x_{j}\right)+\epsilon\left(x_{j}\right)
$$

where $\left\{x_{j}\right\}$ is sampling point and $\epsilon$ is a vector of noise samples.

(2) Add time-jitter error in the above error model

$$
f\left(x_{j}+\delta_{j}\right)+\epsilon\left(x_{j}\right)
$$

where $\left\{\delta_{j}\right\}$ are jitter. Due to the sampling time, points are not met correctly but might differ from the exact ones by not more than a given $\delta$, that is, $\left|\delta_{j}\right| \leq \delta$.

(3) Numerical error in the $n$th iterative step in the iterative algorithm as shown in Theorem 2.1 is $\epsilon_{n}$, that is,

$$
\begin{gathered}
\tilde{f}_{1}=P Q_{X} f+\epsilon_{1}, \\
\tilde{f}_{n+1}=\tilde{f}_{1}+\left(I-P Q_{X}\right)\left(\tilde{f}_{n}\right)+\epsilon_{n+1} .
\end{gathered}
$$

For the first kind of error analysis, it is widely studied, see $[1,3,7,8,10]$. However, the second and third kinds of error analysis have not been given as much attention. The second type of error analysis is presented in band-limited signal spaces [13]. The time-jitter errors without additive noise are studied in $[6,9]$. Some results of the third type of error analysis are shown in reproducing kernel spaces [14]. In this paper, we study the three types of error for the iterative algorithm in shift-invariant spaces.

The paper is organized as follows. In Section 2, we introduce some concepts such as bounded partition of unity, shift-invariant spaces, and the iterative algorithm in shiftinvariant spaces. In Section 3, we give error estimates of the iterative algorithm reconstruction algorithm from noisy samples. 


\section{Notations and Preliminaries}

The shift-invariant spaces under consideration are of the form

$$
V^{2}=\left\{\sum_{k \in Z} c_{k} \psi(\cdot-k):\left\{c_{k}\right\} \in \ell^{2}\right\},
$$

where $\psi$ is the so-called generator. The generator belongs to a subspace of continuous functions of Wiener amalgam spaces $W_{0}\left(L^{1}\right) \subset W\left(L^{1}\right)$. A measurable function $f$ belongs to Wiener amalgam spaces $W\left(L^{1}\right)$ if it satisfies

$$
\|f\|_{W\left(L^{1}\right)}=\sum_{k \in \mathbb{Z}} \text { ess } \sup \left\{|f(x+k)| ; x \in[0,1]^{d}\right\}<\infty
$$

For any $f=\sum_{k \in Z} c_{k} \psi(\cdot-k) \in V^{2}$, we have the norm equivalences $\|f\|_{L^{2}} \approx\|c\|_{\ell^{2}}$ in [3]; that is, there exist constants $\alpha$ and $\beta$ such that

$$
\beta\|f\|_{L^{2}} \leq\|c\|_{\ell^{2}} \leq \alpha\|f\|_{L^{2}}
$$

A set $X=\left\{x_{j}: j \in J\right\}$ satisfying $\inf _{j, l}\left|x_{j}-x_{l}\right|>0$ is called separated, where $J$ is a countable index set. For the sampling set $X$, we can give the following definition of bounded partition of unity (BPU). A bounded partition of unity (BPU) associated with $X=\left\{x_{j}\right\}_{j \in J}$ is a set of functions $\left\{\beta_{j}\right\}$ that satisfy

(1) $0 \leq \beta_{j} \leq 1$, where $j \in J$,

(2) $\operatorname{supp} \beta_{j} \subset B_{\delta}\left(x_{j}\right)$,

(3) $\sum_{j \in J} \beta_{j}=1$.

The operator $Q_{X}$ defined by $Q_{X} f(x)=\sum_{x_{j} \in X} f\left(x_{j}\right) \beta_{x_{j}}(x)$ is a quasi-interpolant of the sampled values $\left.f\right|_{X}$.

Aldroubi and Gröchenig presented the following iterative algorithm in [3].

Theorem 2.1. Let $X=\left\{x_{j}: j \in J\right\}$ be sampling set with $\mathbb{R}^{d}=\bigcup_{j \in J} B_{\delta}\left(x_{j}\right)$ for some $\delta>0$ and $\left\{\beta_{j}\right\}$ be a BPU associated with $X$. Let $\psi$ be in $W_{0}\left(L^{1}\right)$ and $P$ be a bounded projection from $L^{2}\left(\mathbb{R}^{d}\right)$ onto $V^{2}$. Then, there exists density $\delta$ such that if $X=\left\{x_{j}\right\}$ is separated, then any $f \in V^{2}$ can be recovered from its samples $\left\{f\left(x_{j}\right)\right\}_{j \in J}$ on sampling set $X$ by the iterative algorithm

$$
\begin{gathered}
f_{1}=P Q_{X} f, \\
f_{n+1}=P Q_{X}\left(f-f_{n}\right)+f_{n} .
\end{gathered}
$$

\section{Error Estimates of Iterative Reconstruction Algorithm}

First, for given data $\{y\}_{j \in J}$ of the form $y_{j}=f\left(x_{j}\right)+\epsilon\left(x_{j}\right)$, we give the estimation of $\left\|f-f^{\prime}\right\|$ in Theorem 3.3, where $f^{\prime}$ is recovered via the iterative approximation projection reconstruction algorithm in Theorem 2.1 from corrupted samples $\left\{f\left(x_{i}\right)+\epsilon\left(x_{i}\right)\right\}$. If $\epsilon\left(x_{j}\right)$ are noise with zero 
mean and $\sigma^{2}$ variance, then the error estimates of $E\left(f(x)-f^{\prime}(x)\right)$ and $\operatorname{Var}\left(f(x)-f^{\prime}(x)\right)$ are presented in Theorem 3.1. Second, we discuss the second type of error. The estimate of $\mid f(x)-$ $f_{\infty}(x) \mid$ will be shown in Theorem 3.4, where $f_{\infty}$ is recovered via the iterative approximation projection reconstruction algorithm from corrupted samples $\left\{f\left(x_{i}+\delta_{i}\right)+\epsilon\left(x_{i}\right)\right\}$. Third, if there exists the numerical error in $n$th iterative step in the iterative algorithm, then we will consider the numerical stability of the iterative algorithm in Theorem 3.5.

Theorem 3.1. Suppose that the initial samples in the iterative approximation projection reconstruction algorithm as shown by Theorem 2.1 are corrupted, that is, $\left\{f\left(x_{i}\right)+\epsilon\left(x_{i}\right)\right\}$ for noise $\epsilon=\left\{\epsilon\left(x_{i}\right)\right\}$. Assume that $\epsilon(x), x \in X$ are noises with zero mean and $\sigma^{2}$ variance, that is,

$$
E(\epsilon(x))=0, \quad \operatorname{Var}(\epsilon(x))=\sigma^{2} .
$$

If there exists a constant $M>0$ such that

$$
\sum\left|S_{x_{j}}(x)\right|^{2} \leq M
$$

then for any $x \in \mathbb{R}^{d}$

$$
E\left(f(x)-f^{\prime}(x)\right)=0, \quad \operatorname{Var}\left(f(x)-f^{\prime}(x)\right) \leq M \sigma^{2},
$$

where $f^{\prime}$ is recovered via the iterative approximation projection reconstruction algorithm from corrupted samples $\left\{f\left(x_{i}\right)+\epsilon\left(x_{i}\right)\right\}, S_{x_{j}}(x)=\left(I+\sum_{n=1}^{\infty}\left(I-P Q_{X}\right)^{n}\right) P \beta_{x_{j}}(x)$ and $P, Q_{X}$, and $\beta_{x_{j}}$ are defined in Theorem 2.1.

Proof. Applying (2.4) iteratively leads to

$$
f_{n}=\left(I+\sum_{k=1}^{n}\left(I-P Q_{X}\right)^{k}\right) f_{1}(x)
$$

which together with the convergence of (2.4) implies that

$$
f(x)=\sum_{x_{j} \in X} f\left(x_{j}\right) S_{x_{j}}(x),
$$

where $S_{x_{j}}(x)=\left(I+\sum_{n=1}^{\infty}\left(I-P Q_{X}\right)^{n}\right) P \beta_{x_{j}}(x), Q_{X} f(x)=\sum_{x_{j} \in X} f\left(x_{j}\right) \beta_{x_{j}}(x)$.

Combining (3.5) with $E(\epsilon(x))=0$ leads to

$$
E\left(f(x)-f^{\prime}(x)\right)=E\left(\sum_{x_{j} \in X} \epsilon\left(x_{j}\right) S_{x_{j}}(x)\right)=\sum_{x_{j} \in X} E\left(\epsilon\left(x_{j}\right)\right) S_{x_{j}}(x)=0 .
$$


By $\operatorname{Var}(\epsilon(x))=\sigma^{2}$, (3.2), and (3.6), we obtain

$$
\operatorname{Var}\left(f(x)-f^{\prime}(x)\right)=E\left(\sum_{x_{j} \in X} \epsilon\left(x_{j}\right) S_{x_{j}}(x)\right)^{2}=\sigma^{2} \sum_{x_{j} \in X}\left|S_{x_{j}}(x)\right|^{2} \leq M \sigma^{2}
$$

Remark 3.2. If we add some restricted conditions presented in [14] for generator $\psi$, that is,

$$
\left\|\sup _{Z \in \mathbb{R}^{d}}\left|K(\cdot+z, z)\left\|_{L^{1}\left(\mathbb{R}^{d}\right)}<\infty, \quad \lim _{\delta \rightarrow 0}\right\| \sup _{Z \in \mathbb{R}^{d}}\right| \omega_{\delta} K(\cdot+z, z)\right\|_{L^{1}\left(\mathbb{R}^{d}\right)}=0,
$$

where $\omega_{\delta}(f)(x)=\sup _{x^{\prime} \in[-\delta, \delta]}\left|f\left(x+x^{\prime}\right)-f(x)\right|, K(x, y)=\sum_{k \in \mathbb{Z}} \psi(x-k) \tilde{\psi}(y-k)$ and $\{\tilde{\psi}(\cdot-$ $k)\}_{k \in \mathbb{Z}}$ is the dual of $\left.\psi(\cdot-k)\right\}_{k \in \mathbb{Z}}$, then it is easy to check that (3.2) holds in shift-invariant space. Song et al. obtained the restricted conditions such that (3.2) holds for bandlimited space in [15]. From Lemma 2.1 of [16], (3.2) holds for special shift-invariant, that is spline subspace.

Theorem 3.3. If the initial samples in the iterative approximation projection reconstruction algorithm as shown by Theorem 2.1 are corrupted, that is, $\left\{f\left(x_{i}\right)+\epsilon\left(x_{i}\right)\right\}$ for noise $\epsilon=\left\{\epsilon\left(x_{i}\right)\right\}$, then $\left\|f-f^{\prime}\right\|$ is bounded by the $\ell^{2}$ norm of the noise. More precisely,

$$
\left\|f-f^{\prime}\right\| \leq \delta^{1 / 2} \frac{1}{1-\gamma}\|P\|_{o p}\|\| \epsilon \|_{\ell^{2}}
$$

where $f^{\prime}$ is recovered via the iterative approximation projection reconstruction algorithm from corrupted samples $\left\{f\left(x_{i}\right)+\epsilon\left(x_{i}\right)\right\}$ and $\gamma:=\left\|I-P Q_{X}\right\|_{o p}$.

Proof. Let

$$
\begin{gathered}
f_{1}^{\prime}=P Q_{X} f_{1}, \\
f_{n+1}^{\prime}=P Q_{X}\left(f_{1}-f_{n}^{\prime}\right)+f_{n^{\prime}}^{\prime}
\end{gathered}
$$

where $f_{1}=f+\epsilon$.

From the iterative (3.10),

$$
f_{n+1}^{\prime}-f_{n}^{\prime}=\left(I-P Q_{X}\right)\left(f_{n}^{\prime}-f_{n-1}^{\prime}\right)=\cdots=\left(I-P Q_{X}\right)^{n} f_{1}^{\prime} \quad(n \geq 1) .
$$

Using Lemma 8.3 of [3], we may choose $\delta$ so small that

$$
r:=\left\|I-P Q_{X}\right\|_{o p}<1
$$


By (3.4),(3.11), and (3.12), we have

$$
\begin{aligned}
\left\|f_{n}^{\prime}-f\right\|_{2} & \leq \sum_{k=n+1}^{\infty}\left\|\left(I-P Q_{X}\right)^{k}\left(f_{1}^{\prime}-P Q_{X} \epsilon\right)\right\|_{L^{2}}+\sum_{k=0}^{n}\left\|\left(I-P Q_{X}\right)^{k} P Q_{X} \epsilon\right\|_{L^{2}} \\
& \leq \sum_{k=n+1}^{\infty} \gamma^{k}\left\|f_{1}^{\prime}-P Q_{X} \epsilon\right\|_{L^{2}}+\sum_{k=0}^{n} \gamma^{k}\left\|P Q_{X} \epsilon\right\|_{L^{2}} \\
& \leq \sum_{k=n+1}^{\infty} \gamma^{k}\left\|f_{1}^{\prime}\right\|_{L^{2}}+\sum_{k=0}^{\infty} r^{k}\left\|P Q_{X} \epsilon\right\|_{L^{2}} \\
& \leq \frac{1}{1-\gamma}\left(r^{n+1}\left\|f_{1}^{\prime}\right\|_{L^{2}}+\left\|P Q_{X} \epsilon\right\|_{L^{2}}\right) \\
& \leq\|P\|_{o p} \delta^{1 / 2} \frac{1}{1-\gamma}\left(r^{n+1}\left\|\left(f\left(x_{i}\right)+\epsilon\left(x_{i}\right)\right)\right\|_{\ell^{2}}+\left\|\left(\epsilon\left(x_{i}\right)\right)\right\|_{\ell^{2}}\right) .
\end{aligned}
$$

This implies that $\left\|f-f^{\prime}\right\| \leq \delta^{1 / 2} 1 /(1-\gamma)\|P\|_{o p}\|\epsilon\|_{\ell^{2}}$.

Theorem 3.4. Assume that the initial samples in the iterative approximation projection reconstruction algorithm as shown by Theorem 2.1 are corrupted, that is, $\left\{f\left(x_{i}+\delta_{i}\right)+\epsilon\left(x_{i}\right)\right\}$ for noise $\delta=\left\{\delta_{i}\right\}$ with $\sup \left|\delta_{i}\right| \leq \delta$ and $\epsilon=\left\{\epsilon\left(x_{i}\right)\right\}$. If there exist constants $L, M>0$ such that

$$
\sum_{x_{j} \in X}\left|\psi^{\prime}\left(x+x_{j}\right)\right| \leq L<+\infty, \quad \sum\left|S_{x_{j}}(x)\right|^{2} \leq M<+\infty, \quad \forall x \in \mathbb{R}
$$

for the generator $\psi$ and sampling set $X$, then

$$
\left|f(x)-f_{\infty}(x)\right| \leq \sqrt{2 M\left(\alpha^{2} \delta^{2} L^{2}\|f\|^{2}+\sum_{n}\left|\epsilon_{n}\right|^{2}\right)}
$$

where $f_{\infty}$ is recovered via the iterative approximation projection reconstruction algorithm from corrupted samples $\left\{f\left(x_{i}+\delta_{i}\right)+\epsilon\left(x_{i}\right)\right\},\left\{S_{x_{j}}\right\}$ as defined in Theorem 3.1 and $\alpha$ as defined in (2.3).

Proof. From (3.2) and (3.5), we have

$$
\begin{aligned}
\left|f(x)-f_{\infty}(x)\right|^{2} & =\left|\sum_{j}\left[f\left(x_{j}\right)-f\left(x_{j}+\delta_{j}\right)-\epsilon_{j}\right] S_{x_{j}}(x)\right|^{2} \\
& \leq \sum_{j}\left|f\left(x_{j}\right)-f\left(x_{j}+\delta_{j}\right)-\epsilon_{j}\right|^{2} \sum_{j}\left|S_{x_{j}}(x)\right|^{2} \\
& \leq 2 M\left(\sum_{j}\left|f\left(x_{j}\right)-f\left(x_{j}+\delta_{j}\right)\right|^{2}+\sum_{j}\left|\epsilon_{j}\right|^{2}\right) .
\end{aligned}
$$


Next, we will estimate $\sum_{j}\left|f\left(x_{j}\right)-f\left(x_{j}+\delta_{j}\right)\right|^{2}$.

By the Hölder inequality and (3.14),

$$
\begin{aligned}
\sum_{j}\left|f\left(x_{j}\right)-f\left(x_{j}+\delta_{j}\right)\right|^{2} & =\sum_{j}\left|\int_{0}^{\delta_{j}} f^{\prime}\left(t+x_{j}\right) d t\right|^{2} \\
& \leq \sum_{j} \delta_{j} \int_{0}^{\delta_{j}}\left|f^{\prime}\left(t+x_{j}\right)\right|^{2} d t \\
& \leq \delta \int_{0}^{\delta} \sum_{j}\left|f^{\prime}\left(t+x_{j}\right)\right|^{2} d t \\
& =\delta \int_{0}^{\delta} \sum_{j}\left|\sum_{k} c(k) \psi^{\prime}\left(t+x_{j}-k\right)\right|^{2} d t \\
& \leq \delta^{2} L^{2} \sum_{k}|c(k)|^{2} \leq \alpha^{2} \delta^{2} L^{2}\|f\|^{2} .
\end{aligned}
$$

The last inequality follows from (2.3).

So, we have

$$
\begin{aligned}
\left|f(x)-f_{\infty}(x)\right|^{2} & \leq 2\left(\sum_{j}\left|f\left(x_{j}\right)-f\left(x_{j}+\delta_{j}\right)\right|^{2}+\sum_{j}\left|\epsilon_{j}\right|^{2}\right) \sum_{j}\left|S_{x_{j}}(x)\right|^{2} \\
& \leq 2 M\left(\alpha^{2} \delta^{2} L^{2}\|f\|^{2}+\sum_{n}\left|\epsilon_{n}\right|^{2}\right) .
\end{aligned}
$$

Lastly, we will consider the numerical stability of the iterative algorithm.

Theorem 3.5. Assume that the numerical error in nth iterative step in the iterative algorithm as shown in Theorem 2.1 is $\epsilon_{n}$, that is,

$$
\begin{gathered}
\tilde{f}_{1}=P Q_{X} f+\epsilon_{1}, \\
\tilde{f}_{n+1}=\tilde{f}_{1}+\left(I-P Q_{X}\right)\left(\tilde{f}_{n}\right)+\epsilon_{n+1} .
\end{gathered}
$$

Then, we have the following estimation

$$
\left\|f-\tilde{f}_{n}\right\|_{L^{2}} \leq\left(1+\frac{\|P\|_{o p} \delta^{1 / 2}}{1-\gamma}\right)\left((n-1)\left\|\epsilon_{1}\right\|_{L^{2}}+\sum_{j=1}^{n}\left\|\epsilon_{j}\right\|_{L^{2}}\right)+\frac{\gamma^{n}}{1-\gamma}\|P\|_{o p} \delta^{1 / 2}\left\|\left(f\left(x_{j}\right)\right)\right\|_{\ell^{2}},
$$

where $\gamma:=\left\|I-P Q_{X}\right\|_{o p}$. 
Proof. By induction, we obtain $\tilde{f}_{n}-f_{n}=-\sum_{k=1}^{n-1}(I-P Q)^{n-1-k} P Q_{X} \widetilde{\epsilon}_{k}+\widetilde{\epsilon}_{n}$, where $\widetilde{\epsilon}_{1}=\epsilon_{1}$ and $\tilde{\epsilon}_{k}=k \epsilon_{1}+\epsilon_{2}+\cdots+\epsilon_{k}$ for $k \geq 2$.

Using Lemma 8.3 of [3], we may choose $\delta$ so small such that (3.12) holds.

Therefore,

$$
\begin{aligned}
\left\|\tilde{f}_{n}-f_{n}\right\|_{L^{2}}= & \sum_{k=1}^{n-1}\left\|\left(I-P Q_{X}\right)^{n-1-k} P Q_{X} \widetilde{\epsilon}_{k}\right\|_{L^{2}}+\left\|\widetilde{\epsilon}_{n}\right\|_{L^{2}} \\
\leq & \sum_{k=1}^{n-1} \gamma^{n-1-k}\|P\|_{o p} \delta^{1 / 2}\left\|\widetilde{\epsilon}_{k}\right\|_{L^{2}}+\left\|\widetilde{\epsilon}_{n}\right\|_{L^{2}} \\
\leq & \sum_{k=1}^{n-1} \gamma^{n-1-k}\|P\|_{o p} \delta^{1 / 2}\left(k\left\|\epsilon_{1}\right\|_{L^{2}}+\sum_{j=2}^{k}\left\|\epsilon_{j}\right\|_{L^{2}}\right) \\
& +n\left\|\epsilon_{1}\right\|_{L^{2}}+\sum_{j=2}^{n}\left\|\epsilon_{j}\right\|_{L^{2}} \\
\leq & \left(1+\frac{\|P\|_{o p} \delta^{1 / 2}}{1-\gamma}\right)\left((n-1)\left\|\epsilon_{1}\right\|_{L^{2}}+\sum_{j=1}^{n}\left\|\epsilon_{j}\right\|_{L^{2}}\right) .
\end{aligned}
$$

The iterative algorithm converges exponentially too, precisely

$$
\left\|f_{n}-f\right\| \leq \frac{r^{n}}{1-\gamma}\left\|f_{1}\right\|
$$

In fact, by the iterative algorithm

$$
f_{n+1}-f_{n}=\left(I-P Q_{X}\right)\left(f_{n}-f_{n-1}\right)=\cdots=\left(I-P Q_{X}\right)^{n} f_{1}, \quad(n \geq 1) .
$$

From (3.12), we have $\left\|f_{n}-f\right\| \leq \gamma^{n} /(1-\gamma)\left\|f_{1}\right\|$.

Combining (3.21) with (3.22), we have

$$
\begin{aligned}
\left\|f-\tilde{f}_{n}\right\|_{L^{2}} \leq & \left\|\tilde{f}_{n}-f_{n}\right\|_{L^{2}}+\left\|f_{n}-f\right\|_{L^{2}} \\
\leq & \left(1+\frac{\|P\|_{o p} \delta^{1 / 2}}{1-\gamma}\right)\left((n-1)\left\|\epsilon_{1}\right\|_{L^{2}}+\sum_{j=1}^{n}\left\|\epsilon_{j}\right\|_{L^{2}}\right)+\frac{\gamma^{n}}{1-\gamma}\left\|f_{1}\right\| \\
\leq & \left(1+\frac{\|P\|_{o p} \delta^{1 / 2}}{1-\gamma}\right)\left((n-1)\left\|\epsilon_{1}\right\|_{L^{2}}+\sum_{j=1}^{n}\left\|\epsilon_{j}\right\|_{L^{2}}\right) \\
& +\frac{\gamma^{n}}{1-\gamma}\|P\|_{o p} \delta^{1 / 2}\left\|\left(f\left(x_{j}\right)\right)\right\|_{\ell^{2}} .
\end{aligned}
$$




\section{Acknowledgments}

This project is partially supported by the National Natural Science Foundation of China (10801136, 10871213), the Natural Science Foundation of Guangdong Province (07300434), and the Fundamental Research Funds for the Central Universities (10lgpy27). This work is partially done when the author is visiting the Department of Mathematics, University of Central Florida. The author thanks the department for the hospitality. The authors would like to thank Professor Qiyu Sun and Professor Wenchang Sun for their discussion and suggestions.

\section{References}

[1] C. E. Shannon, "Communication in the presence of noise," Proceedings of the IEEE, vol. 37, pp. 10-21, 1949.

[2] A. Aldroubi, C. Leonetti, and Q. Sun, "Error analysis of frame reconstruction from noisy samples," IEEE Transactions on Signal Processing, vol. 56, no. 6, pp. 2311-2325, 2008.

[3] A. Aldroubi and K. Gröchenig, "Nonuniform sampling and reconstruction in shift-invariant spaces," SIAM Review, vol. 43, no. 4, pp. 585-620, 2001.

[4] W. Chen, B. Han, and R.-Q. Jia, "Estimate of aliasing error for non-smooth signals prefiltered by quasi-projections into shift-invariant spaces," IEEE Transactions on Signal Processing, vol. 53, no. 5, pp. 1927-1933, 2005.

[5] B. Liu and W. Sun, "Determining averaging functions in average sampling," IEEE Signal Processing Letters, vol. 14, no. 4, pp. 244-246, 2007.

[6] S. Luo, "Error estimation for non-uniform sampling in shift invariant space," Applicable Analysis, vol. 86, no. 4, pp. 483-496, 2007.

[7] S. Smale and D.-X. Zhou, "Shannon sampling and function reconstruction from point values," American Mathematical Society. Bulletin. New Series, vol. 41, no. 3, pp. 279-305, 2004.

[8] S. Smale and D.-X. Zhou, "Shannon sampling. II. Connections to learning theory," Applied and Computational Harmonic Analysis, vol. 19, no. 3, pp. 285-302, 2005.

[9] W. Sun and X. Zhou, "Sampling theorem for wavelet subspaces: error estimate and irregular sampling," IEEE Transactions on Signal Processing, vol. 48, no. 1, pp. 223-226, 2000.

[10] M. Unser, "Sampling-50 years after Shannon," Proceedings of the IEEE, vol. 88, no. 4, pp. 569-587, 2000.

[11] J. Xian and S. Li, "Sampling set conditions in weighted multiply generated shift-invariant spaces and their applications," Applied and Computational Harmonic Analysis, vol. 23, no. 2, pp. 171-180, 2007.

[12] J. Xian, S.-P. Luo, and W. Lin, "Weighted sampling and signal reconstruction in spline subspaces," Signal Processing, vol. 86, no. 2, pp. 331-340, 2006.

[13] A. Nordio, C.-F. Chiasserini, and E. Viterbo, "Signal reconstruction errors in jittered sampling," IEEE Transactions on Signal Processing, vol. 57, no. 12, pp. 4711-4718, 2009.

[14] M. Z. Nashed and Q. Sun, "Sampling and reconstruction of signals in a reproducing kernel subspace of $L^{p}\left(R^{d}\right)$," Journal of Functional Analysis, vol. 258, no. 7, pp. 2422-2452, 2010.

[15] Z. Song, W. Sun, X. Zhou, and Z. Hou, "An average sampling theorem for bandlimited stochastic processes," IEEE Transactions on Information Theory, vol. 53, no. 12, pp. 4798-4800, 2007.

[16] W. Sun and X. Zhou, "Average sampling in spline subspaces," Applied Mathematics Letters, vol. 15, no. 2, pp. 233-237, 2002. 


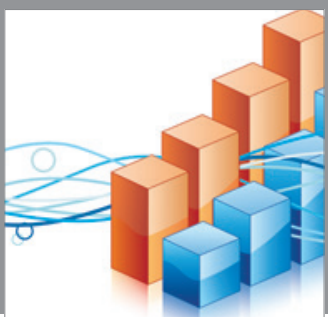

Advances in

Operations Research

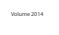

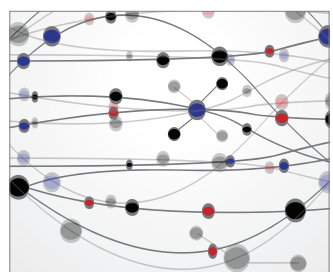

\section{The Scientific} World Journal
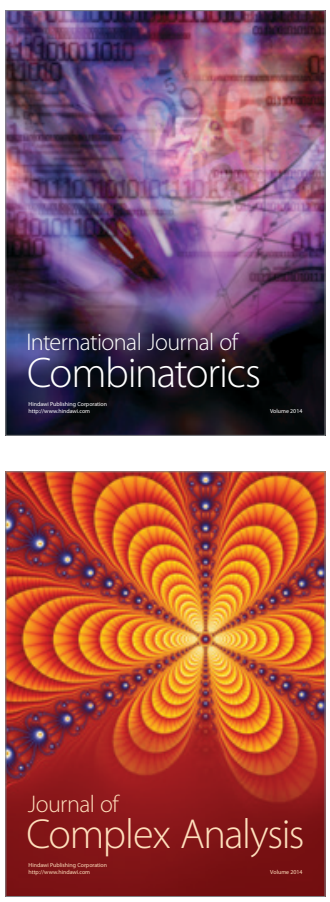

International Journal of

Mathematics and

Mathematical

Sciences
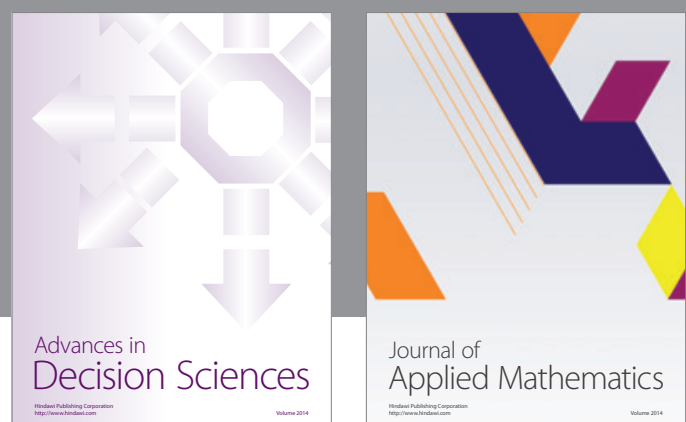

Journal of

Applied Mathematics
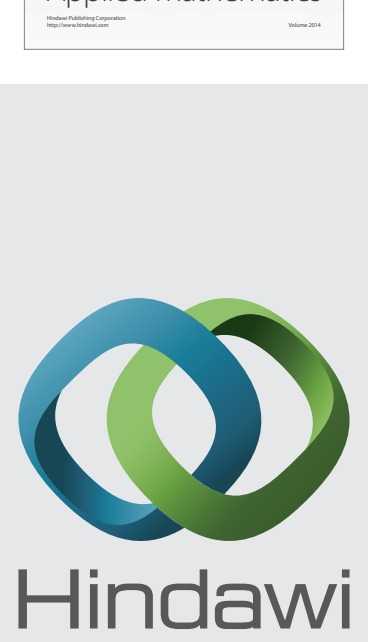

Submit your manuscripts at http://www.hindawi.com
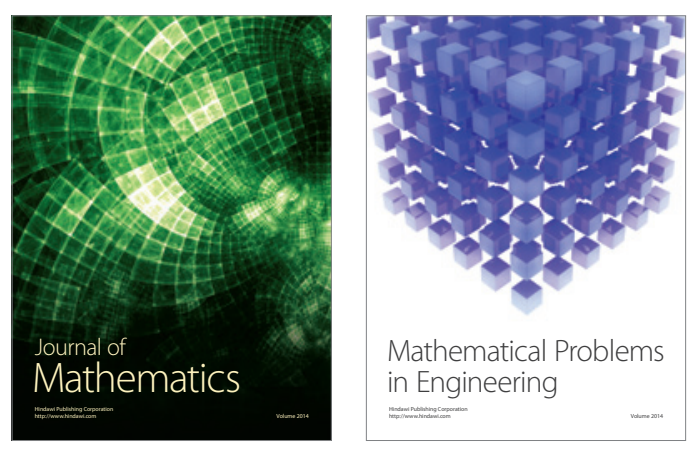

Mathematical Problems in Engineering
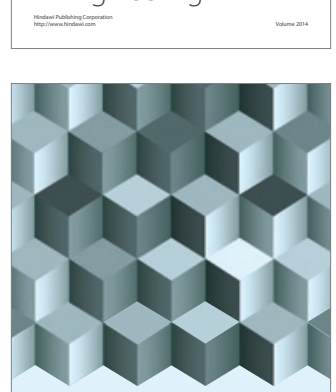

Journal of

Function Spaces
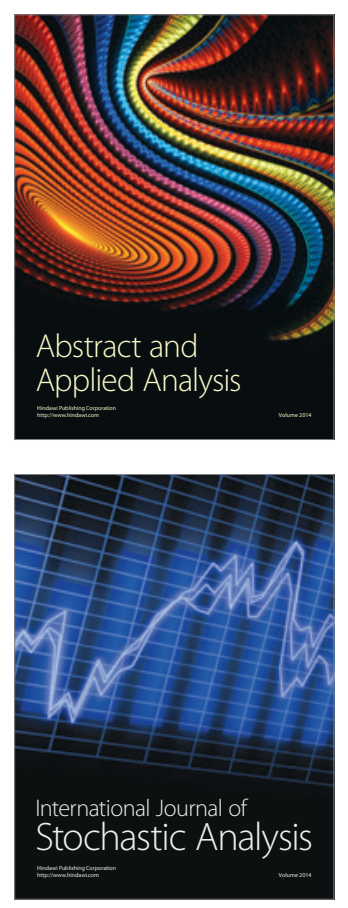

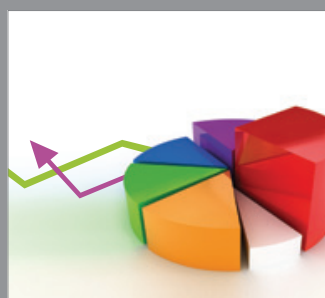

ournal of

Probability and Statistics

Promensencen
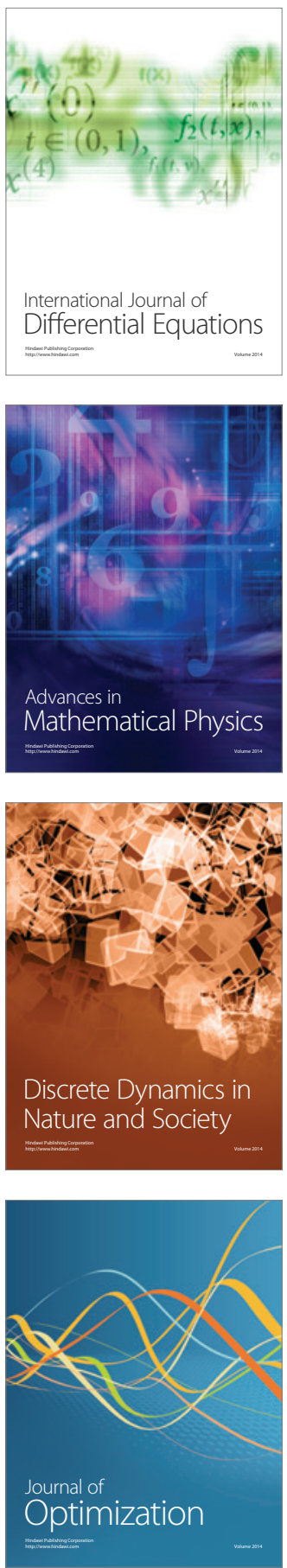